\title{
Autonomous, Distributed Parking Lot Vacancy Management Using Intervehicle Communication
}

\author{
Shohei Yamashita and Kazumasa Takami \\ Graduate School of Engineering, Soka University, 1-236 Tangi-cho, Hachioji-shi 192-8577, Japan \\ Correspondence should be addressed to Kazumasa Takami; k_takami@soka.ac.jp
}

Received 25 March 2014; Accepted 20 May 2014; Published 21 July 2014

Academic Editor: Martin Reisslein

Copyright ( 2014 S. Yamashita and K. Takami. This is an open access article distributed under the Creative Commons Attribution License, which permits unrestricted use, distribution, and reproduction in any medium, provided the original work is properly cited.

\begin{abstract}
We have developed a method of managing vacancy information of a large parking lot in a distributed manner using only intervehicle communication. A group of parking spaces is defined as a cluster. Vacancy information of a cluster is managed by a vehicle in it. This vehicle is called a cluster head. The proposed method generates a communication path topology between cluster heads. The topology is a tree structure with the cluster head of the cluster nearest to the parking lot entrance as the root node. Cluster heads are ranked in order of the number of vacant spaces and the distance to the shop entrance. The vehicle entering the parking lot collects vacancy information of clusters. This information is transmitted along the tree structure from the lowest ranking cluster head. We have developed a simulation model for a parking lot that can accommodate nearly 1,000 vehicles and used it to evaluate the proposed method. We have confirmed that the proposed method generates less communication traffic and enables the vehicle entering a parking lot to collect vacancy information about the area near the shop entrance with a higher probability and in a shorter time.
\end{abstract}

\section{Introduction}

Today, large commercial facilities invariably have large parking lots. For example, the parking lot of Tokyo Disney Resort [1] accommodates 20,000 vehicles, and that of representative shopping mall in Japan 4,000 vehicles. There are more than 150 theme parks in Japan that have parking lots for 1,000 or more vehicles. If vehicles are concentrated on a specific area in a parking lot, such as the areas near shop entrances, they jam these areas and generate traffic congestion, causing drivers to waste time and lose incentive to do shopping. This means lost opportunities for both shoppers and shop owners.

A system that is attracting interest as a means of solving this problem is intelligent transport systems (ITS) [2, 3]. ITS addresses a range of traffic issues, such as traffic optimization, by linking drivers, roads, and vehicles using leadingedge telecommunication technology. It mainly uses roadvehicle communication and intervehicle communication. Road-vehicle communication is used in the electronic toll collection (ETC) system and the vehicle information and communication system (VICS) in Japan. ETC uses dedicated short-range communication (DSRC) for communication between the road and the vehicle. Intensive studies are being made on intervehicle communication that conforms to ITS FORUM RC-005 to 007 [3] and IEEE802.11p $[4,5]$. Intervehicle communication is expected to prevent accidents at sites where the view of the road ahead is blocked, and so on [613]. Although it is not yet widely used, its feasibility tests are actively conducted. Most applications that use intervehicle communication target prevention of accidents, and few have been proposed for use for business purposes.

There is a study in which an access network is formed using road-vehicle communication and intervehicle communication. Vacancy information of a parking lot is collected by using the access network and centrally managed by a server [14]. An access point is installed in the parking lot. When a vehicle parks, it informs the server of its location via the access point using intervehicle communication. Based on this information, the server notifies a vehicle entering the parking lot of the availability of parking spaces. In another study, a wireless sensor is installed at each parking space and a server manages vacancy information [15]. When a wireless sensor detects that a vehicle has parked at the parking space it 
covers, it informs the server of it via a wireless sensor network. Still another study uses only road-vehicle communication $[16,17]$. A number of access points are installed in a parking lot in such a way that they will cover the entire parking lot. When a vehicle parks, the information about its location is sent to the server via an access point and is centrally managed there. There is also a proposal to centrally manage vacancy information of multiple parking lots in a city and to calculate the probability of successful parking in order to recommend parking lots with vacant parking spaces one by one [18]. However, all of these proposals incur costs of installing and operating a dedicated server, access points, or wireless sensors.

This paper proposes a method of managing vacancy information of a parking lot in an automatic, distributed manner by equipping vehicles with server and intervehicle communication capabilities. The method has been evaluated by simulation. Section 2 outlines the proposed method and identifies issues to be studied. Section 3 proposes solutions to these issues. Section 4 discusses the experimental system developed and the evaluation of the proposed solutions. Section 5 presents conclusions and future issues.

\section{Autonomous, Distributed Parking Lot Vacancy Management}

The authors propose to manage vacancy information of a parking lot in an autonomous, distributed manner using only intervehicle communication. No server, wireless access points, or wireless sensors are required. Two alternative methods can be conceived for the transmission and management of vacancy information. (1) Each time a vehicle enters the parking lot, all parked vehicles send its current parking space information such as the parking section and location number. (2) A vehicle near the entrance to the parking lot centrally manages vacancy information. A problem with (1) is that a large volume of traffic arises because each time a vehicle makes a request for entry, all vehicles have to establish communication paths and send data. A problem with (2) is the lack of impartiality because one vehicle (near the entrance) consumes far greater power than other vehicles. To solve these problems, we propose an autonomous, distributed management of vacancy information. In this method, a group of vehicles are defined as a cluster, and one of the vehicles in a cluster is designated as the "cluster head $(\mathrm{CH})$." The $\mathrm{CH}$ manages all the other vehicles (called "child vehicles") in the cluster and communicates with other CHs.

The method is outlined in Figure 1. The parking lot is divided into sections. Vehicles in a section form a cluster. When a vehicle enters a parking lot, it communicates with CHs. The driver can get vacancy information of even those sections that are hidden from his/her view. An item of vacancy information of a cluster consists of the name and location of the cluster and the number of parking spaces occupied. The vacancy information is shown on the monitor of the car navigation system, and so forth, as shown in Figure 1. However, for this method to be highly effective, it is necessary that all the vehicles involved have an intervehicle communication capability. The proposed service assumes that all the vehicles involved will have an intervehicle communication capability in the future.

The intervehicle communication protocol stack assumed in this paper is shown in Figure 2. The proposed vacancy management method is provided at the application layer. The network layer is assumed to be a simple packet carry-andforward protocol. In addition, this paper does not discuss capabilities of the network layer, functions allocation between the network layer and the application layer.

The issues that need to be solved to implement the proposed method are the following.

Issue 1: Cluster Forming Method. It is necessary to study how to form clusters in a way that will enable distributed management of vacancy information.

Issue 2: Topology That Defines the Relations between Cluster Heads. It is necessary to study the topology of the paths for passing messages between $\mathrm{CH}$ s to convey vacancy information.

Issue 3: Method of Collecting Information and Types of Information to Be Provided. After the above topology has been determined, it is necessary to study what types of information to send and how to send them to the vehicle that has entered the parking lot. Unless the information is sent just in time, packets may be sent in vain. If the parking lot is large, the volume of data to send can also become large. It is necessary to minimize the volume of data transmitted.

\section{Solutions}

3.1. Algorithm for Forming a Cluster. The parking lot is divided into sections, and the parking spaces in each section are treated as a cluster. Figure 3 shows examples of a cluster and the cluster management information held by the cluster head $(\mathrm{CH})$. How a particular parking lot is divided and ranked is predetermined. The ID and location of each cluster are preregistered in the car navigation systems of the patrons of the parking lot. The first vehicle that has come to a cluster is designated as the $\mathrm{CH}$. The $\mathrm{CH}$ manages the vehicle ID and the parking space location number of other vehicles (child vehicles) that have come to that section. The parking space location number is painted on the floor in advance and is captured by a vehicle-mounted camera. The parking space location number can be replaced by GPS location information. When the $\mathrm{CH}$ leaves the parking lot, another vehicle in the cluster takes over as the $\mathrm{CH}$. Three types of messages are defined to form a cluster:

vehicle inquiry message (cluster ID, sender ID): a message sent by a vehicle that has come to a section to inquire about the presence of a vehicle,

$\mathrm{CH}$ information message (sender ID, destination ID): a message sent by the $\mathrm{CH}$ in response to a vehicle inquiry message to notify the sender that it is the parent vehicle $(\mathrm{CH})$, 

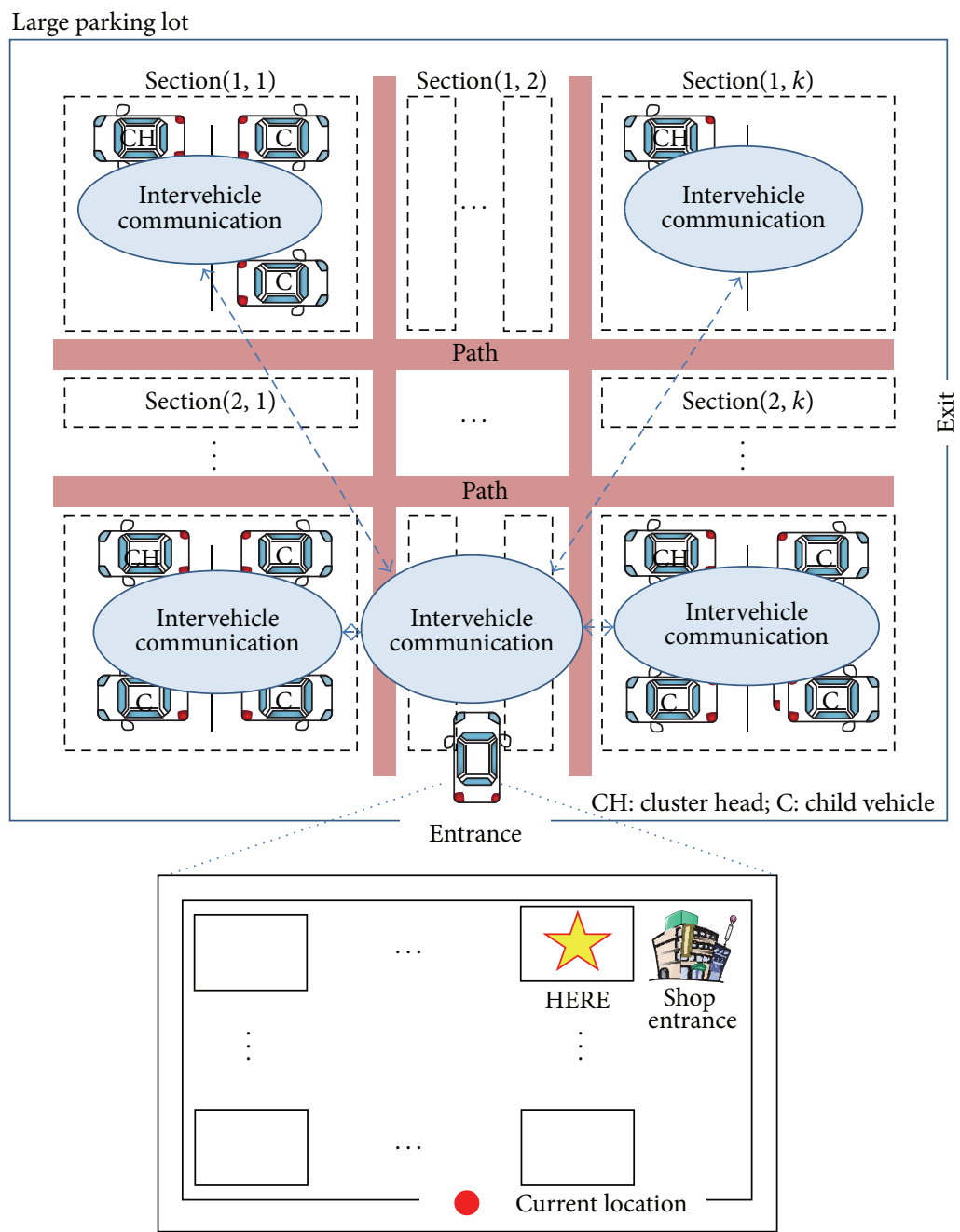

Example of a window displayed on a car navigation system monitor

FIgURE 1: Conceptual diagram of the proposed system.

exit message (cluster ID, sender ID, vacancy information, succeeding $\mathrm{CH}$ ID, and topology information): a message sent by the $\mathrm{CH}$ when it leaves the parking lot.

Messages are sent in one of two ways: broadcasting a message and sending a message to a receiver specified by the cluster number or destination ID in the message. When a vehicle arrives at a section, it broadcasts a vehicle inquiry message. The $\mathrm{CH}$ that has received the message checks the cluster ID in the message. If the cluster ID is the same as its own cluster ID, the $\mathrm{CH}$ saves the sender ID in the message and sends back a $\mathrm{CH}$ information message that contains its own vehicle ID. If the vehicle that has sent the vehicle inquiry message receives a $\mathrm{CH}$ information message within a certain period, it becomes a child vehicle of that cluster. If it does not, it determines that there is no vehicle in that cluster and designates itself as the $\mathrm{CH}$. When a vehicle is to exit the parking lot, it broadcasts an exit message containing its vehicle ID to the other vehicles in the cluster. When the $\mathrm{CH}$ is to leave the parking lot, the succeeding
$\mathrm{CH}$ is selected from the vehicles in the cluster and receives the management information from the exiting $\mathrm{CH}$. It then updates the management information and becomes the $\mathrm{CH}$.

3.2. Algorithm for Forming a Communication Path Topology. Generally three types of topology can be considered as candidates for representing relations between CHs: tree, star, and mesh. However, none of these is suitable for representing the hierarchy and topology of each $\mathrm{CH}$ rank. Besides, they assume that a $\mathrm{CH}$ near the entrance directly communicates with all the $\mathrm{CH}$ to gather vacancy information. This can increase the processing load of that $\mathrm{CH}$. We use a tree structure to represent the message exchange relations between $\mathrm{CHs}$ because this structure is compatible with the hierarchy management that is based on the ranking of $\mathrm{CHs}$ and thus allows efficient collection of vacancy information.

Two types of messages are defined to form the topology:

topology request message (cluster ID, sender ID, and TTL (time to live)): a message broadcast by a vehicle 


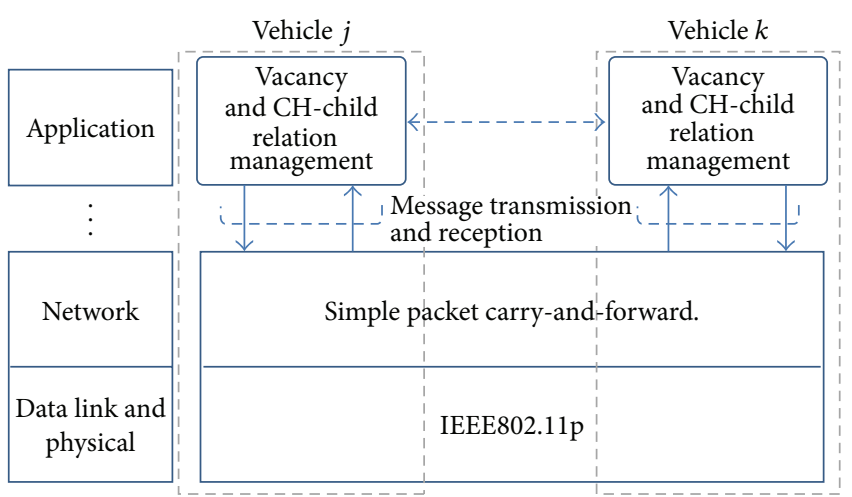

CH: cluster head

Figure 2: Example of the assumed intervehicle communication protocol stack.

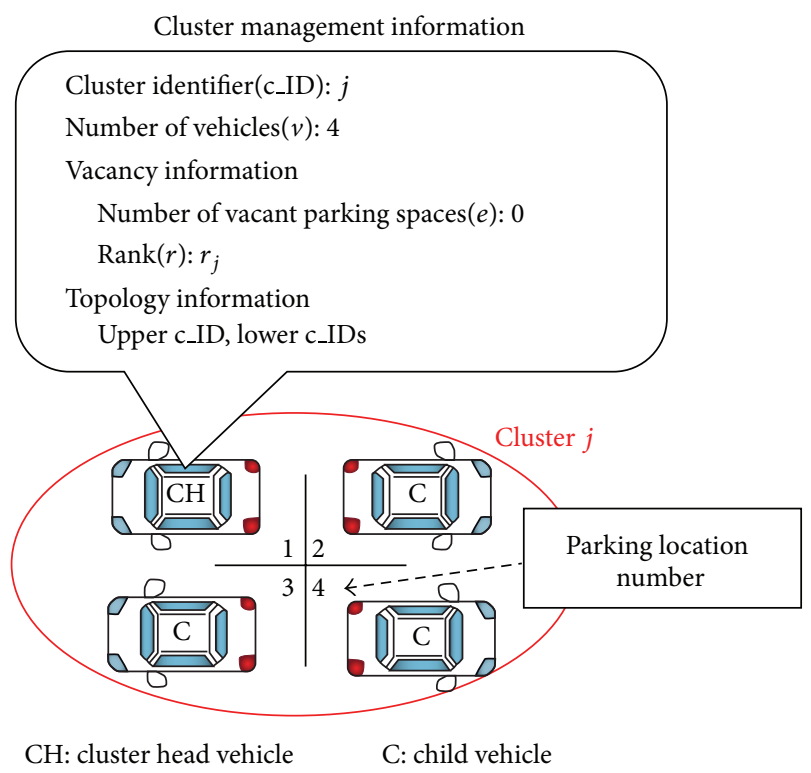

Figure 3: Example of the cluster management information held by the $\mathrm{CH}$ vehicle.

near the parking lot entrance when a topology is to be formed,

lower $\mathrm{CH}$ information message (cluster ID, destination ID, and sender ID): a message sent by a $\mathrm{CH}$ in response to a topology request message.

A vehicle near the parking lot entrance broadcasts a topology request message. A $\mathrm{CH}$ that has received this message saves the sender ID. If the sender ID is the same as that in a topology request message received earlier, the $\mathrm{CH}$ does nothing. If the sender ID is not the same and if the TTL value is greater than 0 , the $\mathrm{CH}$ broadcasts a topology request message and overwrites the existing sender ID with it. It also sends a lower $\mathrm{CH}$ information message in which the sender ID is its own vehicle ID and the destination ID is the saved sender ID. This enables both $\mathrm{CHs}$ to recognize the vehicle ID of each other. A topology is formed through successive transmissions of topology request messages and lower $\mathrm{CH}$ information messages.

There are reports on studies regarding the routing of a MANET (mobile ad hoc network) based on the topology between clusters $[19,20]$. These studies focus on the mobility (moving speed and direction) of each node in a MANET. In particular, the topologies used for routing between $\mathrm{CHs}$ are designed to allow efficient routing by predicting the movements of CHs. Our study also allows a hierarchy to be built by treating nearby $\mathrm{CHs}$ as neighboring $\mathrm{CHs}$. This can be done by making $\mathrm{CHs}$ exchange their location information and calculate distances between CHs. However, in this paper, $\mathrm{CHs}$ stand still. Since the geographic relations between $\mathrm{CHs}$ are based on the division of a parking lot, they are much clearer than those of freely moving nodes in a MANET. Therefore, in this paper, the topology of $\mathrm{CHs}$ is built using a simple algorithm. As the parking lot size increases, so does the number of $\mathrm{CHs}$. This increases the number of messages flooded and consequently affects the scalability of the proposed algorithm. The effect of the increase in the number of messages flooded can be minimized by using a small value for TTL.

3.3. Algorithm for Collecting Vacancy Information Using Ranking of Clusters in Order of the Distance to the Shop Entrance. Two types of messages are defined for information collection:

vacancy information request message (sender ID): a message sent by a vehicle or the $\mathrm{CH}$ near the parking lot entrance to request vacancy information,

vacancy information message (sender ID, destination ID, cluster ID, and vacancy information): a message that contains vacancy information being managed by the sender and is sent by a $\mathrm{CH}$ in response to a vacancy information request message.

If every $\mathrm{CH}$ should send a vacancy information message, a large volume of data would be transmitted. Taking into consideration the fact that drivers tend to prefer to park near the shop entrance, this algorithm attempts to reduce the amount of data transmitted by giving priority to the transmission of vacancy information of sections near the shop entrance.

For the above purpose, sections are ranked in order of the distance to the shop entrance. Concentric zones are defined with the shop entrance at the center. The zone nearest to the center is rank 0 ; the next zone is rank 1 , and so forth. The longer the distance, the larger the rank value. Figure 4 shows an example of the topology of the relations between $\mathrm{CHs}$ and of ranks of CHs. Figure 5 shows the message exchange sequence for collecting vacancy information.

The detailed algorithm for collecting vacancy information is as follows. Its flowchart is shown in Figure 6.

Step 1. A vehicle that has come to the parking lot entrance broadcasts a vacancy information request message. $\mathrm{CH}_{1}$ (root $\mathrm{CH}$ ), that has received this message and is the nearest to the entrance, sends a vacancy information request message to $\mathrm{CH}_{2}$ and $\mathrm{CH}_{3}$ based on the tree structure formed by the algorithm described in Section 3.2. 


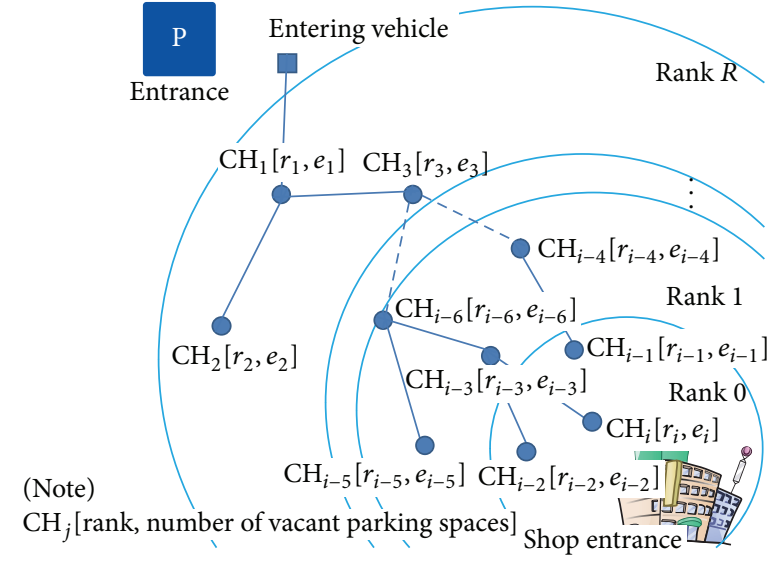

FIGURE 4: Example of a tree structure and assignment of ranks.

Step 2. When $\mathrm{CH}_{3}$ has received the vacancy information request message, it sends a vacancy information request message to the lower $\mathrm{CHs}$ in the tree structure. It waits for a vacancy information message from the lower $\mathrm{CHs}$ for a waiting period of $T[s]$. The waiting period gradually decreases as a vacancy information request propagates to the lower CHs. Consider

Waiting period $T$

$$
=\text { (predefined number of hops }
$$

- the number of hops up to its own position)

$$
\times \Delta t
$$

Step 3. After $T$ [s] has elapsed, $\mathrm{CH}_{i-2}, \mathrm{CH}_{i-1}$, and $\mathrm{CH}_{i}$ send the number of vacant parking spaces, the rank, and cluster ID of their clusters to the upper $\mathrm{CHs}$, that is, $\mathrm{CH}_{i-4}$ and $\mathrm{CH}_{i-3}$.

Step 4. $\mathrm{CH}_{i-3}$ receives the number of vacant parking spaces, the rank, and cluster ID from $\mathrm{CH}_{i-2}$ and $\mathrm{CH}_{i}$, respectively. It compares the rank received with its own rank and selects the one that has the lowest rank. As the ranks of $\mathrm{CH}_{i-2}$ and $\mathrm{CH}_{i}$ are the same, if $e_{i-2}>e_{i}$, then $\mathrm{CH}_{i-3}$ selects $\mathrm{CH}_{i-2}$ because $\mathrm{CH}_{i-2}$ has more vacant parking spaces than $\mathrm{CH}_{i}$. If both $\mathrm{CH}_{i-2}$ and $\mathrm{CH}_{i}$ have the same number of vacant parking spaces, one of them is selected at random.

Step 5. $\mathrm{CH}_{i-3}$ sends the rank and the number of vacant parking spaces of $\mathrm{CH}_{i-2}$ to the upper $\mathrm{CH}$.

When the above process is repeated, the vacancy information of clusters near the shop entrance reaches the entering vehicle without causing a large amount of data to be transmitted. For example, if the number of clusters is 10 , the rank is assigned $0 \sim 2$, respectively, and the number of vacant parking spaces of $\mathrm{CH}$ is shown as Figure 7 , the entering vehicle receives the vacancy information of $\mathrm{CH}_{8}$. "The predefined number of hops" used in the waiting period is predefined for each parking lot. It is the maximum height (i.e., the maximum number of hops) of the tree in the parking lot. The expression (predefined number of hops-the number of hops of its own position) is used to ensure that the larger the number of hops, the shorter the waiting period of the entering vehicle. In other words, the farther a $\mathrm{CH}$ is from the vehicle near the parking lot entrance, the earlier it sends a vacancy information message after it received a vacancy information request message. $\Delta t$ is a parameter that represents the interval of message transmissions.

\section{Experimental System and Evaluation}

4.1. Building an Experimental System. To simulate communication in the above parking lot model, we customized Scenargie [21], a traffic flow simulator, and used it to evaluate the proposed method and a broadcast method. In the latter method, clusters are not defined and no tree structure is formed. To obtain vacancy information, an entering vehicle broadcasts a request message, which contains TTL, to all parked vehicles. A vehicle that has received the request message broadcasts its parking location. It also decrements TTL in the request message by 1 and broadcasts the request message. After this process has been repeated, the entering vehicle receives the vehicle location numbers of parked vehicles, and its driver selects a vacant parking space near the shop entrance based on the received information.

The cluster forming algorithm, the communication path topology forming algorithm, and the information collection algorithm mentioned in Section 3 were implemented in the simulator. The simulation conditions used are shown in Table 1. Ranks are segmented for each $100 \mathrm{~m}$. The maximum rank is set to 5 when the number of parking spaces in a parking lot is 256,8 when the number of parking spaces is 576,9 when the number of parking spaces is 784, and 11 when the number of parking spaces is 1,024 .

\subsection{Evaluation Results}

(1) Generated Tree Structure. A part of the tree structure generated in our experiment is shown in Figure 8. The relationship between the number of nodes and the tree height of the topology is shown in Table 2. Figure 8 shows that the tree structure originates at the vehicle nearest to the parking lot entrance at the upper left corner. Since messages were transmitted at random, CHs were not necessarily linked with the shortest links. Figure 8 shows that almost all vehicles were covered by the topology. An effect of random transmissions of messages is that the upper $\mathrm{CH}$ of Cluster $\mathrm{C}$ was not $\mathrm{A}$ but B. Table 2 shows that, in the case where the number of nodes was 800 and the distance from the parking lot entrance to the shop entrance was about $1130 \mathrm{~m}$, a tree with a height of 10 with few detours was generated. The average maximum number of hops was 9. The larger the maximum number of hops is, the longer it takes to receive information from far vehicles. Therefore, it takes a long time for a vehicle near the parking 


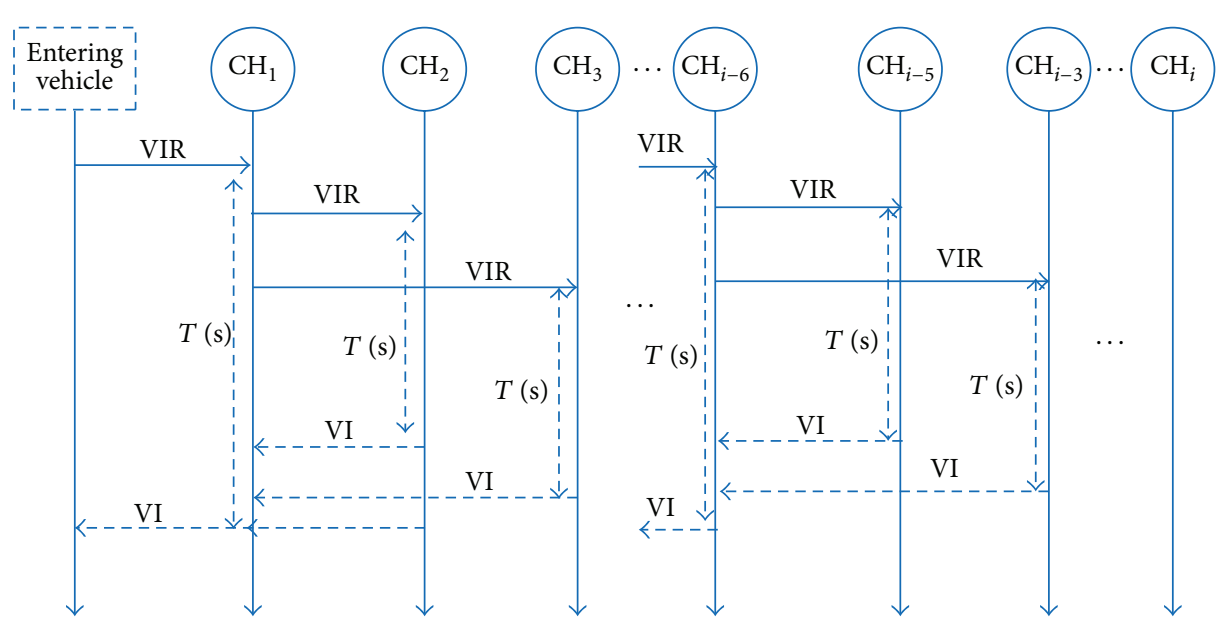

VIR: vacancy information request, VI: vacancy information

FIGURE 5: Example of message sequence for information collection.
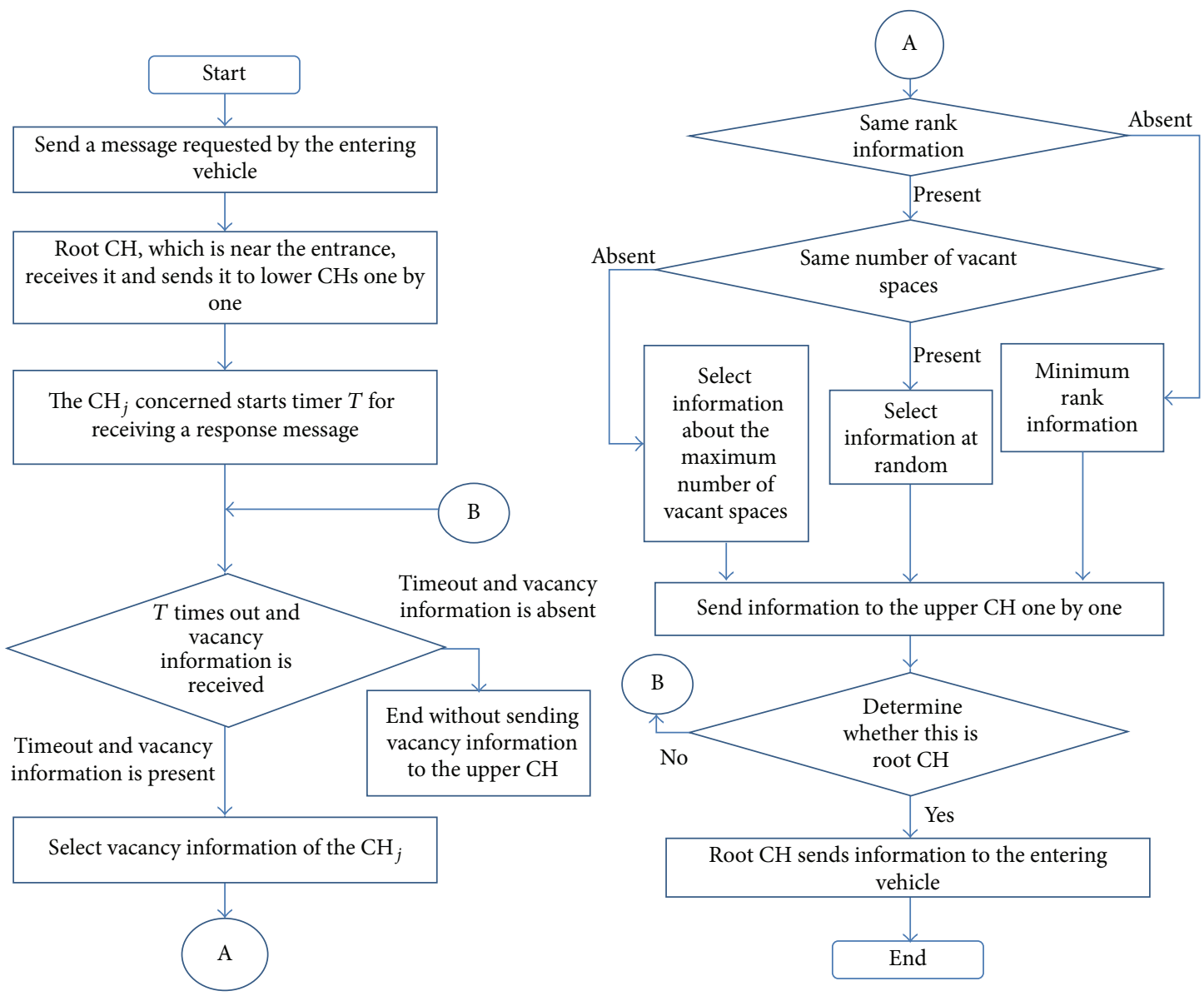

FIGURE 6: Flowchart of the vacancy information gathering algorithm.

lot entrance to receive vacancy information of clusters near the shop entrance.

(2) Topology Forming Time and Topology Forming Ratio. Figure 9 shows the topology forming time, which is the time it takes to form a topology for all the $\mathrm{CHs}$. The proportion of $\mathrm{CHs}$ that are covered by the formed topology is referred to as the "topology forming ratio." These are defined in the following:

Topology forming time

$=[$ Time when a vehicle received the last topology response message] 


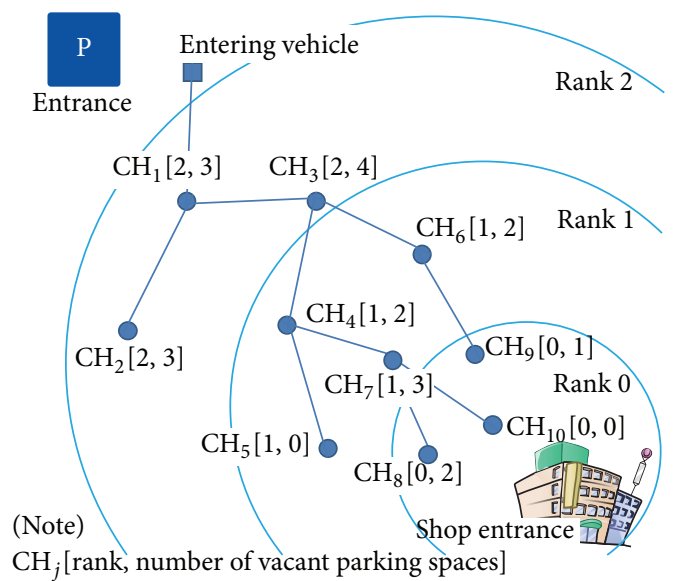

Figure 7: Example of the tree structure that $\mathrm{CHs}$ are assigned the rank and the number of vacant parking spaces.

TABLE 1: Simulation conditions.

\begin{tabular}{|c|c|}
\hline Simulation software & Scenargie $[21]$ \\
\hline Number of nodes (vehicles) & $200,400,600,800$ \\
\hline Area size & $\begin{array}{ll}400 \times 400 & 600 \times 600 \\
700 \times 700 & 800 \times 800\end{array}$ \\
\hline $\begin{array}{l}\text { Radius of the area covered by the } \\
\text { radio wave }\end{array}$ & $110 \mathrm{~m}$ \\
\hline $\begin{array}{l}\text { Number of parking spaces in a } \\
\text { cluster }\end{array}$ & 4 \\
\hline $\begin{array}{l}\text { Number of parking spaces in a } \\
\text { parking }\end{array}$ & $256,576,784,1024$ \\
\hline Rank & $\begin{array}{cc}0 \sim 5 & 0 \sim 8 \\
0 \sim 9 & 0 \sim 11\end{array}$ \\
\hline Wireless LAN specification & IEE802.11p \\
\hline Transmission interval: $\Delta t$ & $0.5,1.0,1.5,2.0 \mathrm{~s}$ \\
\hline Number of experiments & 5 \\
\hline
\end{tabular}

- [Time when a vehicle near the entrance sent a topology request message (i.e., lower $\mathrm{CH}$ information message)].

Topology forming ratio

$=[$ Number of those nodes for which

the upper $\mathrm{CH}$ and the lower

$\mathrm{CH}$ know each other's vehicle ID]

$\div$ [Number of all nodes]

Figure 9 shows that the topology forming time was more or less proportional to the number of nodes. The reason why the topology forming time differed greatly between the case where the number of nodes was 200 and the case where it was 400 can be explained as follows. When the number of nodes was 200, the packet loss due to the receiving buffer

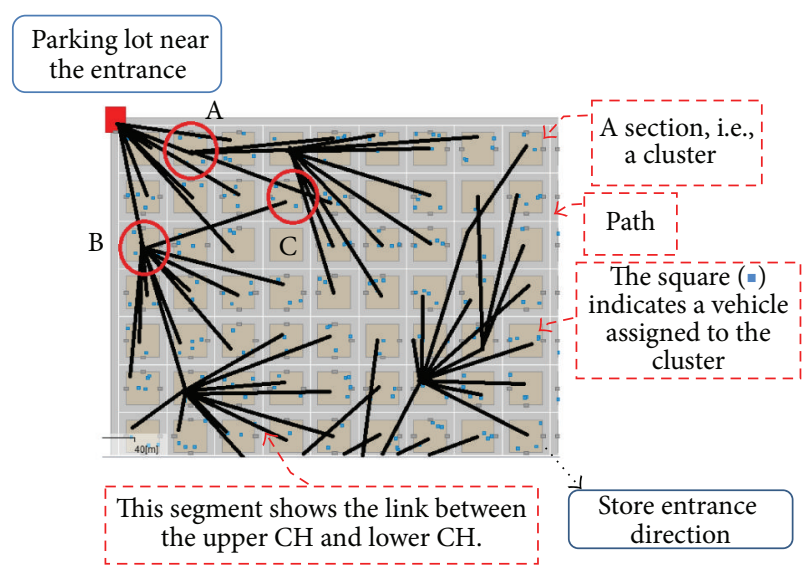

FIGURE 8: A part of generated topology (number of nodes $=800$ ).

TABLE 2: Number of nodes (vehicles) and the height of the generated tree.

\begin{tabular}{lcccc}
\hline Number of nodes & 200 & 400 & 600 & 800 \\
Tree height & 6 & 7 & 8 & 10 \\
\hline
\end{tabular}

overflow was small, and thus almost all vehicles were able to receive messages by the second transmission. In contrast, when the number of vehicles was 400 or more, some vehicles received messages in the third transmission. The reason why the forming time did not vary greatly when the number of nodes increased beyond 400 was that the increase in the forming time was mostly due to an increase in the tree height. From these results, we can deduce that the topology forming time depends on the transmission interval and that it can be reduced by either reducing the transmission interval, $\Delta t$, or reducing the number of transmissions. The topology forming ratio was $99 \%$ or higher irrespective of the number of vehicles. Since all the vehicles received a topology request message, the main reason why the ratio was not $100 \%$ can be that some packets were lost.

(3) Response Ratio and the Obtained Vacancy Information. Figure 10 shows the relationship between the response ratio and the elapsed time for vacancy information collection. Table 3 shows vacancy information received by a vehicle near the parking lot entrance in the case where the number of nodes was 800 . The response ratio is defined by the following:

$$
\begin{aligned}
& \text { Response ratio } \\
& =\text { (number of nodes that returned a vacancy } \\
& \quad \text { information message }) \\
& \quad \div \text { (number of all } \mathrm{CHs})
\end{aligned}
$$

Figure 10 shows that $95 \%$ or more nodes responded before 16 seconds elapsed. Irrespective of the number of nodes, the response ratio rose sharply after several seconds. This indicates that there were more nodes in the middle and high ranks than in the lower rank. If the response ratio is high, and if information can be collected from the lower $\mathrm{CHs}$ 
TABLE 3: Received vacancy information (number of nodes $=800$ ).

\begin{tabular}{lcccc}
\hline 1st time & 2nd time & 3rd time & 4th time & 5th time \\
\hline Rank 0; 3 vacant spaces & Rank 0; 3 vacant spaces & Rank 1; 1 vacant space & Rank 1; 3 vacant spaces & Rank 0;2 vacant spaces \\
\hline
\end{tabular}

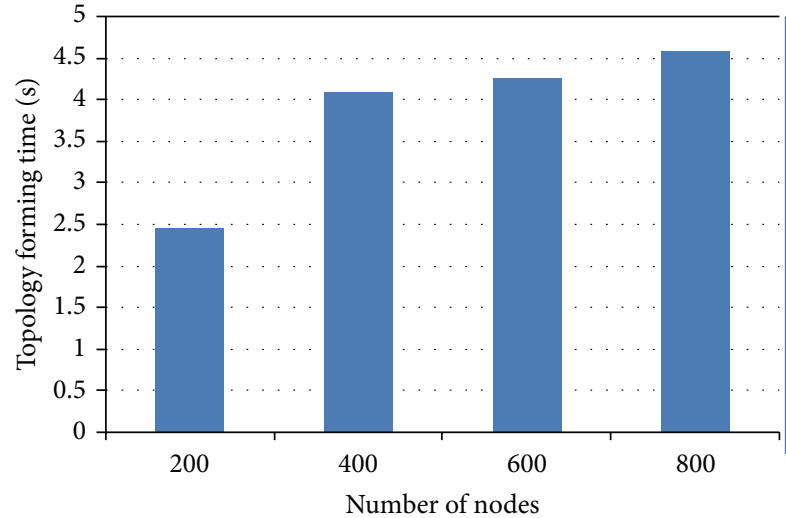

FIGURE 9: Relationship between the number of nodes (vehicles) and the topology forming time.

within the waiting period of each $\mathrm{CH}$, the probability at which a vehicle can collect vacancy information about the area near the shop entrance is high. Table 3 confirms that, while the maximum rank was 11, information about the area of rank 1 or 0 , which was near the shop entrance, was successfully collected. In this way, the time it takes for a vehicle near the parking lot entrance to obtain information about the area near the shop entrance increases as the size of the parking lot increases. However, the packet traffic generated was lower than that with the broadcast method, and the response ratio was as high as nearly $100 \%$.

(4) Waiting Period and Collected Vacancy Information. Figure 11 shows the relationship between the waiting period, $T$, and the rank from which vacancy information was collected for the case where the number of vehicles was 800 . The waiting period is the elapsed time from the time when a vacancy information request message was sent to the time when a vacancy information message about the area nearest to the shop entrance was received. Figure 11 indicates that, in the case where $\Delta t$ was below 1 second, the average rank from which vacancy information was collected was 3.8 and that, in the case where $\Delta t$ was 1 second or longer, the average rank was below 1 . This can be explained as follows. In the case where $\Delta t$ was below 1 second, the waiting period was short, but the cluster in which to park was selected before vacancy information from lower $\mathrm{CHs}$ was collected. As a result, vacancy information about the area near the shop entrance was not transmitted.

\section{Conclusions and Future Issues}

We have proposed a method of managing vacancy information of a large parking lot in an autonomous, distributed manner and evaluated this method by simulation using

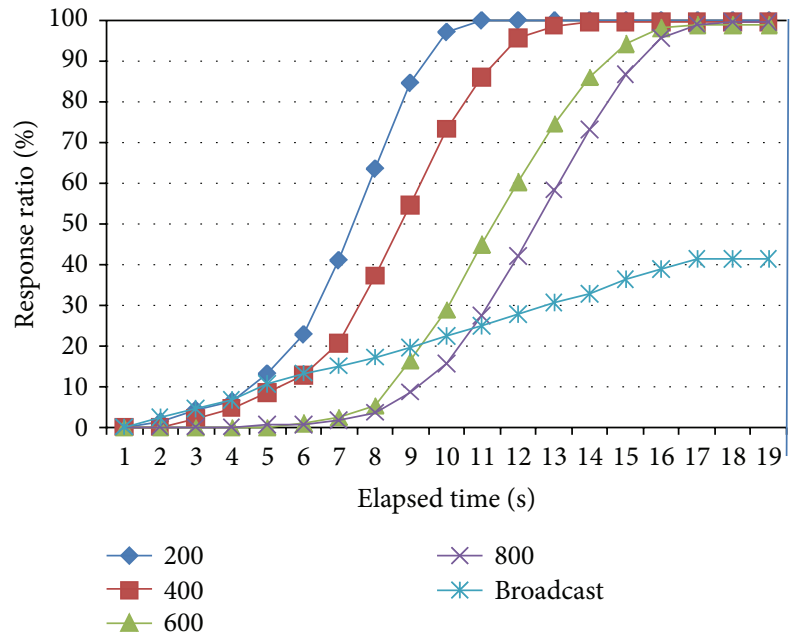

FIGURE 10: Relationship between the response ratio and the elapsed time for vacancy information collection.

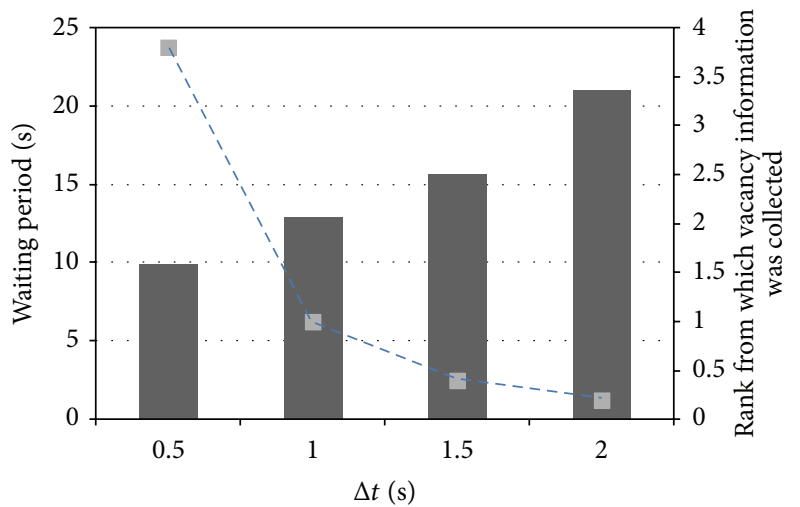

Waiting period

Rank from which vacancy information was collected

FIGURE 11: Relationship between the rank from which vacancy information was collected and the transmission interval, $\Delta t$.

Scenargie. The method forms a tree-structured communication path topology between parking space clusters. We have found that the proposed method does not generate a large amount of traffic, enables responses to a message inquiring about vacancy to be received from $99 \%$ of all parked vehicles within about 16 seconds, and enables a vehicle entering a parking lot to get vacancy information about the area near the shop entrance. These results confirm the effectiveness of the proposed method. It will be necessary to improve the algorithms used in the proposed method in order to take vehicle mobility into consideration, reduce the topology forming time, reduce the maximum number of 
hops, evaluate power consumption of vehicles, and evaluate cases where there is more than one entrance to the parking lot. In particular, to reduce power consumption of $\mathrm{CH}$ s that stay parked over a long period, it is necessary to study the possibility of rotating $\mathrm{CHs}$ periodically.

\section{Conflict of Interests}

The authors declare that there is no conflict of interests regarding the publication of this paper.

\section{References}

[1] “The parking lot of the Tokyo Disney resort,” May 2012, http://ja .wikipedia.org/wiki.

[2] "Intelligent Transport Systems (ITS)," Road Bureau Ministry of Land, Infrastructure, Transport and Tourism, Japan, http:// www.mlit.go.jp/road/ITS/j-html/.

[3] “ITS Info-communications Forum," 2014, http://www.itsforum .gr.jp/E_index.html.

[4] D. Eckhoff, C. Sommer, and F. Dressler, "On the necessity of accurate IEEE 802.11P models for IVC protocol simulation," in Proceedings of the IEEE 75th Vehicular Technology Conference (VTC Spring '12), pp. 1-5, Yokohama, Japan, June 2012.

[5] C. Campolo, A. Cortese, and A. Molinaro, "CRaSCH: a cooperative scheme for service channel reservation in 802.11p/WAVE vehicular ad hoc networks," in Proceedings of the International Conference on Ultra Modern Telecommunications \& Workshops ( ICUMT '09), Saint-Petersburg, Russia, October 2009.

[6] V. Namboodiri and L. Gao, "Prediction-based routing for vehicular Ad Hoc networks," IEEE Transactions on Vehicular Technology, vol. 56, no. 4, pp. 2332-2345, 2007.

[7] T. Kosch, C. J. Adler, S. Eichler, C. Schroth, and M. Strassberger, "The scalability problem of vehicular ad hoc networks and how to solve it," IEEE Wireless Communications, vol. 13, no. 5, pp. 2228, 2006.

[8] M. Raya and J.-P. Hubaux, "Securing vehicular ad hoc networks," Journal of Computer Security, vol. 15, no. 1, pp. 39-68, 2007.

[9] F. Borgonovo, A. Capone, and M. Cesana, "RR-ALOHA, a reliable R-ALOHA broadcast channel for ad-hoc inter-vehicle communication networks," in Proceedings of the Med-Hoc-Net, pp. 15-19, 2002.

[10] Y. Zang, L. Stibor, B. Walke, H. Reumerman, and A. Barroso, "A novel MAC protocol for throughput sensitive applications in vehicular environments," in Proceedings of the IEEE 65th Vehicular Technology Conference (VTC '07), pp. 2580-2584, April 2007.

[11] R. M. Yadumurthy, A. Chimalakonda, M. Sadashivaiah, and R. Makanaboyina, "Reliable MAC broadcast protocol in directional and omni-directional transmissions for vehicular ad hoc networks," in Proceedings of the 2nd ACM International Workshop on Vehicular Ad Hoc Networks (VANET '05), pp. 1019, September 2005.

[12] I. Broustis and M. Faloutsos, "Routing in vehicular networks: feasibility, modeling, and Security," International Journal of Vehicular Technology, vol. 2008, Article ID 267513, 8 pages, 2008.

[13] S. Kamakshi and S. Natarajan, "Distributed algorithm for constructing efficient tree topology for message dissemination in vehicular networks," International Journal of Vehicular Technology, vol. 2014, Article ID 903895, 9 pages, 2014.

[14] K. Matsukawa, "Parking Position Management System and Parking Position Management Method," 2014, http://patent .astamuse.com/ja/granted/JP/No/4696016.

[15] W. S. Vanessa, Y. Zheng, and J. Cao, "An intelligent car park management system based on wireless sensor networks," in Proceedings of the 1st International Symposium on Pervasive Computing and Applications, pp. 65-70, August 2006.

[16] R. Lu, X. Lin, H. Zhu, and X. Shen, "SPARK: a new VANETbased smart parking scheme for large parking lots," in Proceedings of the IEEE INFOCOM, pp. 1413-1421, Rio de Janeiro, Brazil, April 2009.

[17] R. Lu, X. Lin, H. Zhu, and X. Shen, "An intelligent secure and privacy-preserving parking scheme through vehicular communications," IEEE Transactions on Vehicular Technology, vol. 59, no. 6, pp. 2772-2785, 2010.

[18] E. H.-K. Wu, J. Sahoo, C.-Y. Liu, M.-H. Jin, and S.-H. Lin, “Agile urban parking recommendation service for intelligent vehicular guiding system," IEEE Intelligent Transportation Systems Magazine, vol. 6, no. 1, pp. 35-49, 2014.

[19] Y. Wang, T. Liang, X. Yang, and D. Zhang, "Scalable and effective cluster based routing algorithm using nodes' location for Mobile Ad Hoc Networks," Information Technology Journal, vol. 7, no. 7, pp. 958-971, 2008.

[20] L. Ritchie, H. Yang, A. W. Richa, and M. Reisslein, "Cluster Overlay Broadcast (COB): MANET routing with complexity polynomial in source-destination distance," IEEE Transactions on Mobile Computing, vol. 5, no. 6, pp. 653-666, 2006.

[21] Scenargie, "Space-Time Engineering," https://www.spacetimeeng.com/en/index.html. 

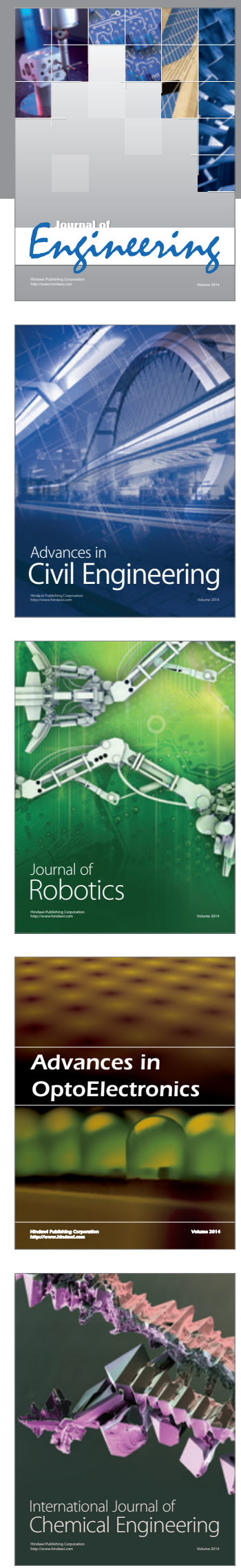

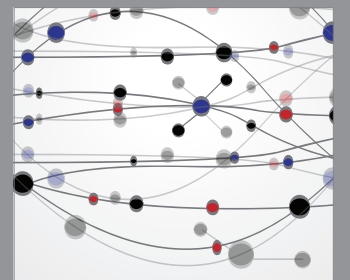

The Scientific World Journal
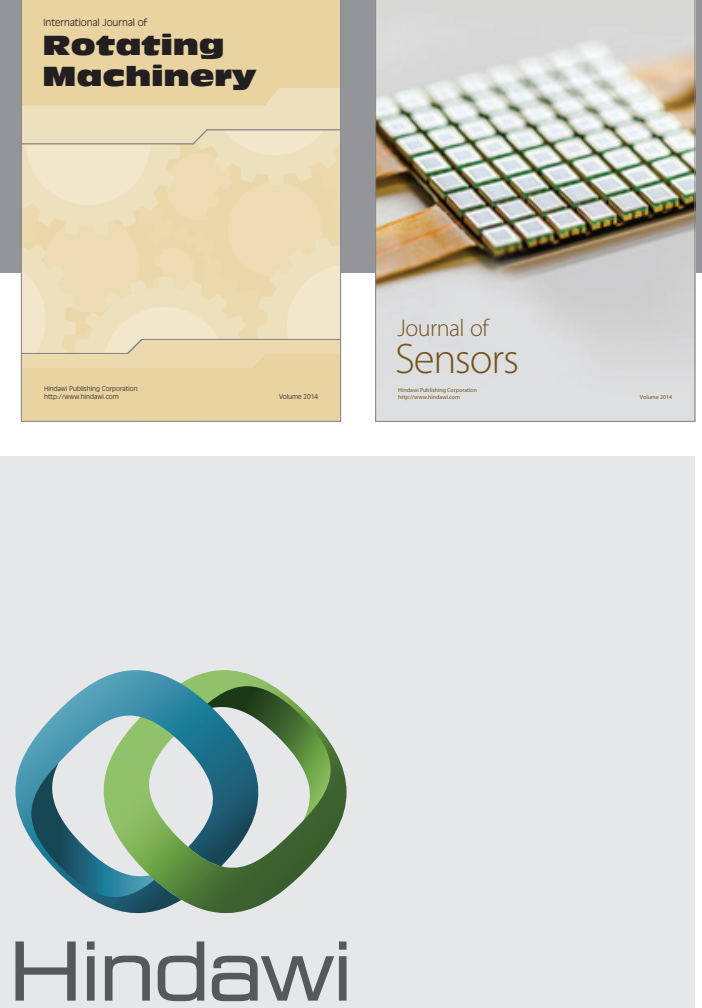

Submit your manuscripts at http://www.hindawi.com
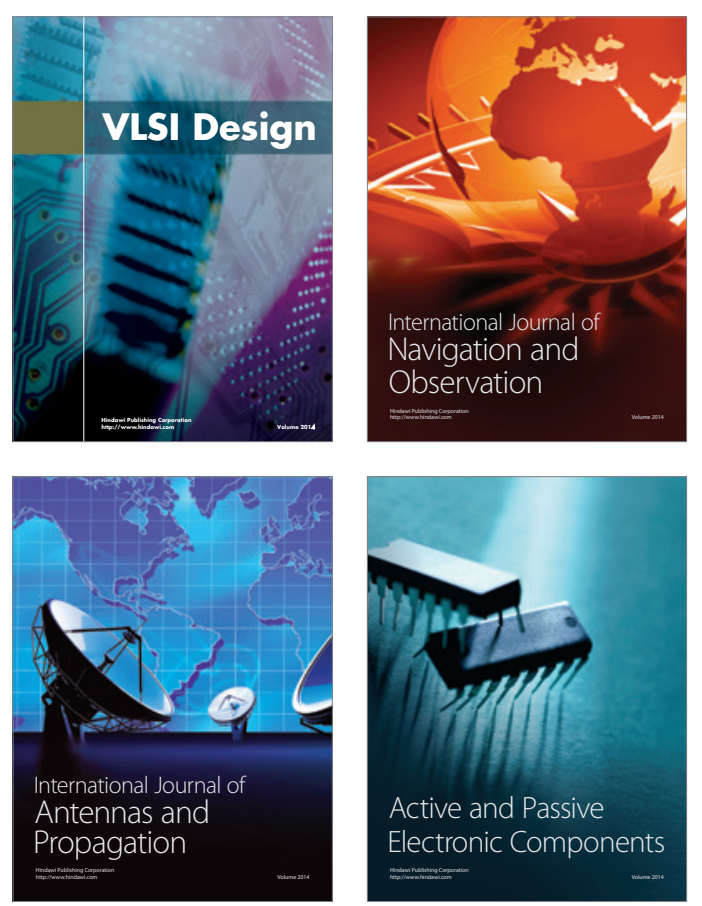
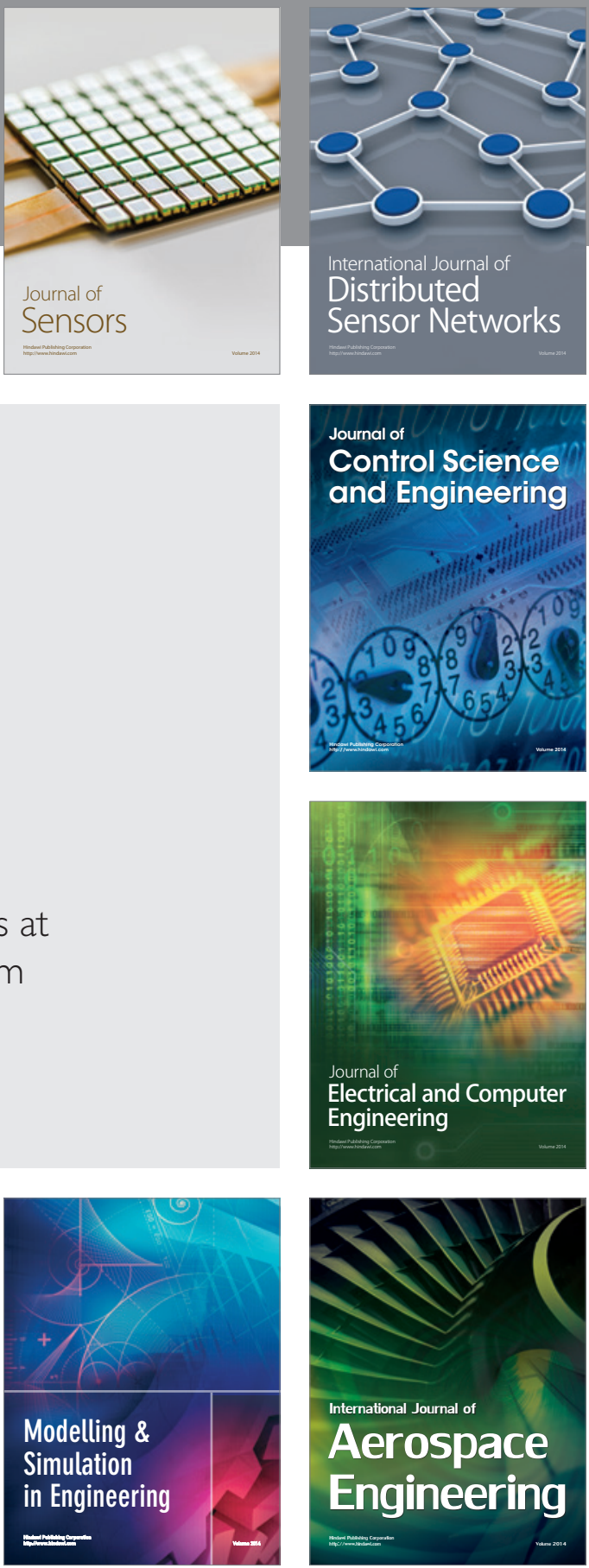

Journal of

Control Science

and Engineering
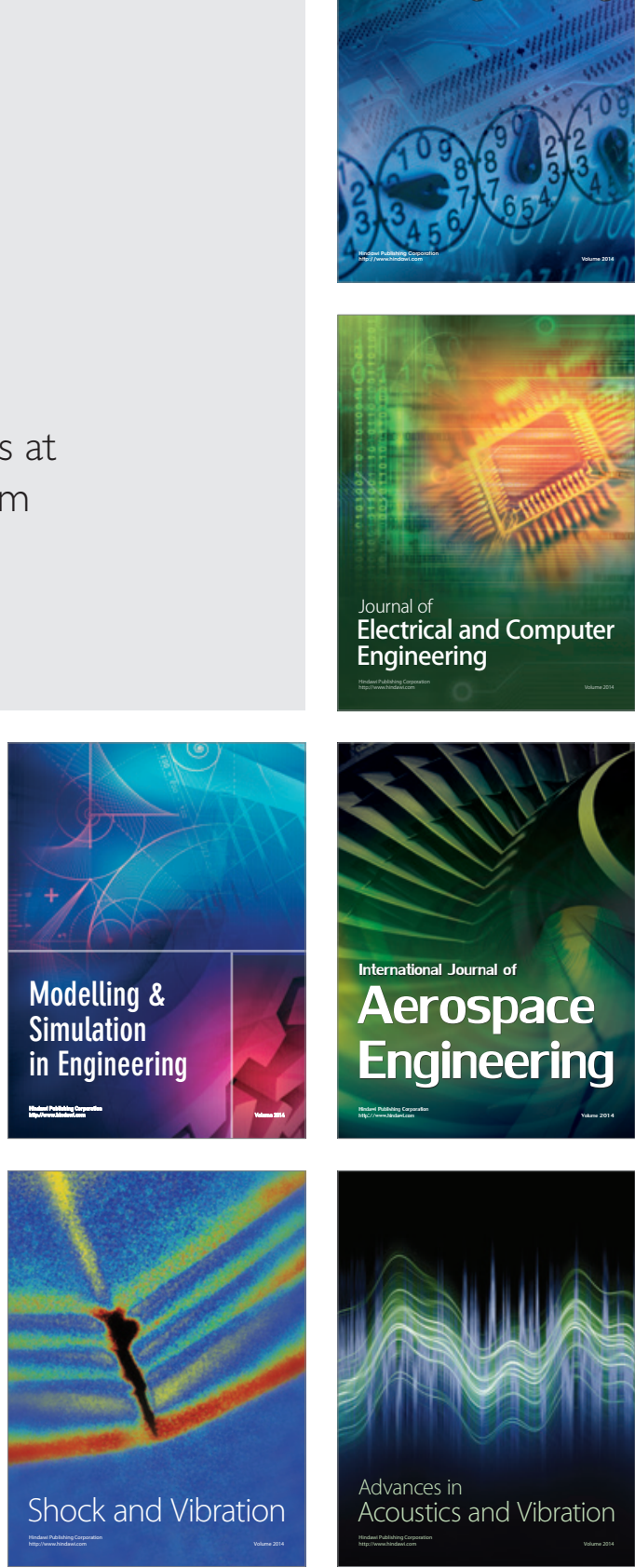